EPJ Web of Conferences 71, 00083 (2014)

DOI: 10.1051/epjconf/20147100083

(C) Owned by the authors, published by EDP Sciences, 2014

\title{
$B$-Physics and Quarkonia at the CMS experiment
}

\author{
Luca Martini ${ }^{1,2, a}$ for the CMS collaboration \\ ${ }^{1}$ Università di Pisa \\ ${ }^{2}$ INFN sez. di Pisa
}

\begin{abstract}
The most recent results on $B$-Physics and Quarkonia physics at the CMS experiment are presented. The focus of the talk is on the charmonia polarization, a search for new bottomonium states and the measurement of the $B_{s}^{0} \rightarrow \mu^{+} \mu^{-}$branching fraction.
\end{abstract}

\section{Introduction}

The CMS collaboration is actively involved in the measurements of many relevant quantities related to quarkonia and $B$-physics. The latest publications on this field, up to the conference date, are on the measurement of the prompt charmonia polarization, on the search for a new resonance decaying into the $\Upsilon(1 \mathrm{~S})$ meson and on the first measurement of the $B_{s}^{0} \rightarrow \mu^{+} \mu^{-}$branching fraction.

\section{Prompt $J / \psi$ and $\psi(2 S)$ meson polarizations}

Despite decades of theoretical and experimental research, quarkonium production is not yet well understood. In fact, the nonrelativistic quantum chromodynamics (NRQCD) does not satisfactorily describes the small longitudinal polarization of $J / \psi$ mesons, as measured by the CDF collaboration. Since the measurement includes both directly produced $J / \psi$ mesons and those coming from feed-down decays of heavier charmonia (like $\psi(2 S)$ and $\chi_{c}$ ), the comparison between the theoretical predictions and the experimental measurements could be ambiguous.

In this aspect, the measurement of the polarization of the $\psi(2 S)$ meson could be more appealing: given the absence of feed-down from heavier charmonia, a more direct comparison to theoretical calculations is feasible.

The polarization of $J^{\mathrm{PC}}=1^{--}$quarkonium states can be described through their di-lepton decay, with three angular distribution parameters $\vec{\lambda}=\left(\lambda_{\vartheta}, \lambda_{\varphi}, \lambda_{\vartheta \varphi}\right)$. Considering the polar and azimuthal angles of the positive muon, $\vartheta$ and $\varphi$, with respect to the $z$ axis of the chosen polarization frame, the angular distribution can be written as

$$
W(\cos \theta, \varphi \mid \vec{\lambda})=\frac{3}{4 \pi\left(3+\lambda_{\vartheta}\right)}\left(1+\lambda_{\vartheta} \cos ^{2} \vartheta+\lambda_{\varphi} \sin ^{2} \vartheta \cos 2 \varphi+\lambda_{\vartheta \varphi} \sin 2 \vartheta \cos \varphi\right)
$$

An other further important parameter is the frame-invariant polarization parameter $\tilde{\lambda}=\left(\lambda_{\vartheta}+3 \lambda_{\varphi}\right) /(1-$ $\left.\lambda_{\varphi}\right)$.

\footnotetext{
a e-mail: luca.martini@ cern.ch
} 

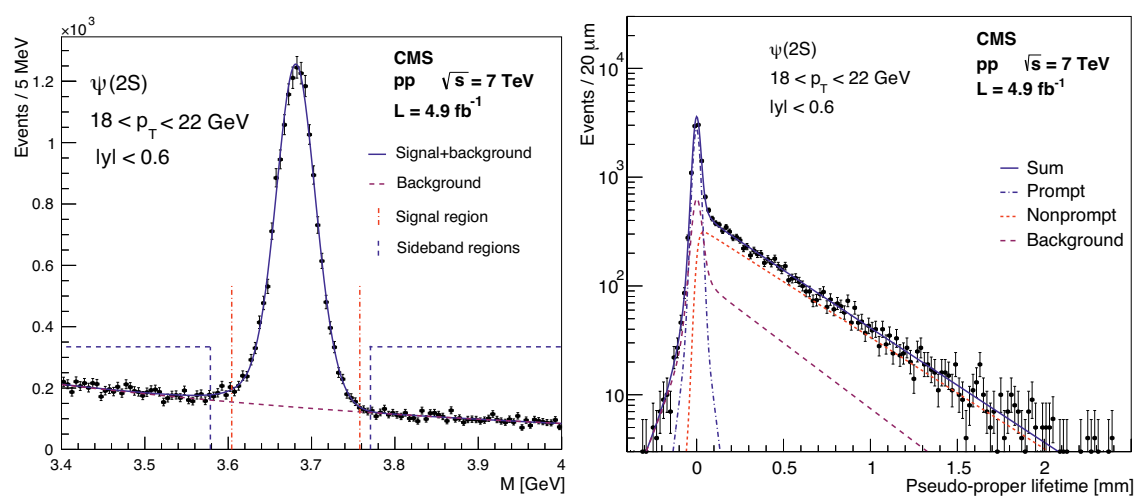

Figure 1. Dimuon invariant-mass (left) and lifetime (right) distributions in the $\psi(2 S)$ mass region for $18<p_{T}<$ $22 \mathrm{GeV}$ and $|y|<0.6$. The result of the fit is shown by the solid curve, representing the sum of three contributions: prompt $\psi(2 S)$ (dash-dotted), nonprompt $\psi(2 S)$ (dotted), and background (dashed).

The CMS collaboration performed the measurements of $\vec{\lambda}$ and $\tilde{\lambda}$ in three polarization frames to obtain a robust angular description of the process [1]. The three reference frames are the following:

- the helicity frame (HX), in which the $z$ axis coincides with the direction of the quarkonium momentum;

- the Collins-Soper frame (CS), where the $z$ axis is the bisector of the two beam directions in the charmonium rest frame;

- the perpendicular helicity frame (PX), with the $z$ axis orthogonal to that in the CS frame.

The $y$ axis is along the vector product of the two beam directions in the charmonium rest frame.

This analysis uses $p p$ collision data collected in the 2011 LHC run, at a centre-of-mass energy of $\sqrt{s}=7 \mathrm{TeV}$, corresponding to an integrated luminosity of $4.9 \mathrm{fb}^{-1}$. The $J / \psi$ meson $\vec{\lambda}$ parameters are measured in several bins of transverse momentum $p_{T}$, in the range $14-70 \mathrm{GeV}$, and in two bins of absolute rapidity $|y|$. For the $\psi(2 S)$ meson measurements are performed in three $y$ bins, in the range $14-50 \mathrm{GeV}$. The dimuons are reconstructed by combining two well-reconstructed opposite-sign muon tracks.

Candidate events passing the trigger and off-line selections are extracted with an unbinned maximum likelihood fit to the dimuon invariant mass. The nonprompt component, mostly from decays of $B$ mesons, is removed by using the dimuon pseudo-proper lifetime $\ell=L_{x y} \cdot m_{\psi(n \mathrm{~S})} / p_{T}$ where $L_{x y}$ is the transverse decay length in the laboratory frame (Fig. 1). The modeling uses the per-event error provided by the vertex reconstruction algorithm. The prompt signal region is measured to be in the range $12-25 \mu \mathrm{m}$, improving with increasing dimuon $p_{T}$.

The background-subtracted events are then used to calculate the posterior probability density of the prompt meson polarization parameters $\vec{\lambda}$, for each bin:

$$
\mathcal{P}(\vec{\lambda})=\prod_{i} \mathcal{E}\left(\vec{p}_{1}^{(i)}, \vec{p}_{2}^{(i)}\right)
$$

where $\mathcal{E}$ is the probability density as a function of the two muon momenta $\vec{p}_{1,2}^{(i)}$ in event $i$ :

$$
\mathcal{E}\left(\vec{p}_{1}, \vec{p}_{2}\right)=\frac{1}{\mathcal{N}(\vec{\lambda})} W(\cos \vartheta, \varphi \mid \vec{\lambda}) \epsilon\left(\vec{p}_{1}, \vec{p}_{2}\right)
$$



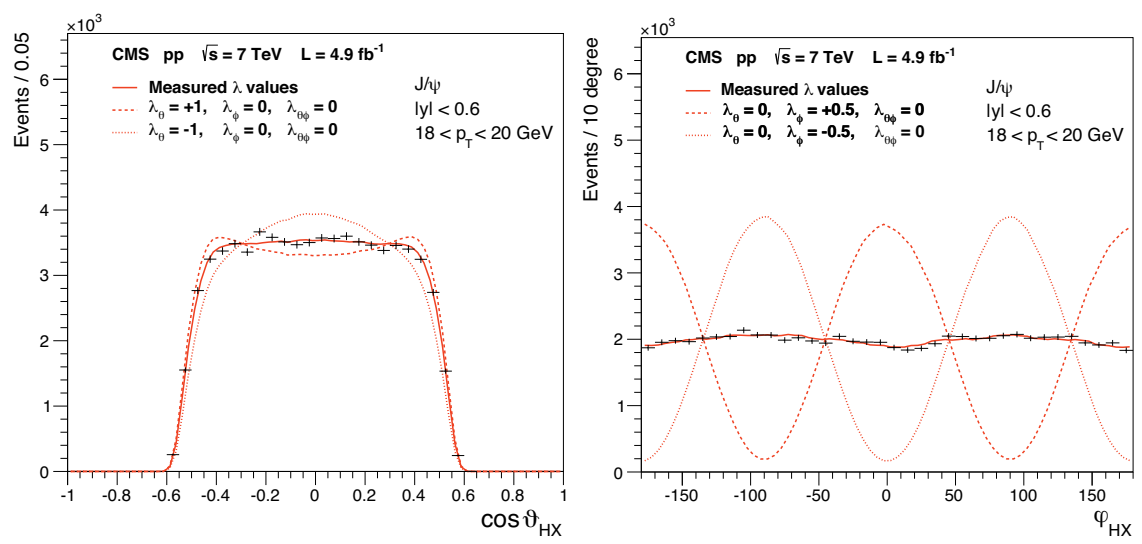

Figure 2. Frequency distributions of the $\cos \vartheta$ (left) and $\varphi$ (right) angular variables, in the HX frame for the $J / \psi$ meson in an intermediate $p_{T}$ bin and in $|y|<0.6$. The curves represent the expected distributions for two extreme polarization scenarios (dashed and dotted lines defined in the legends) and for the measured $\vec{\lambda}$ (solid lines).
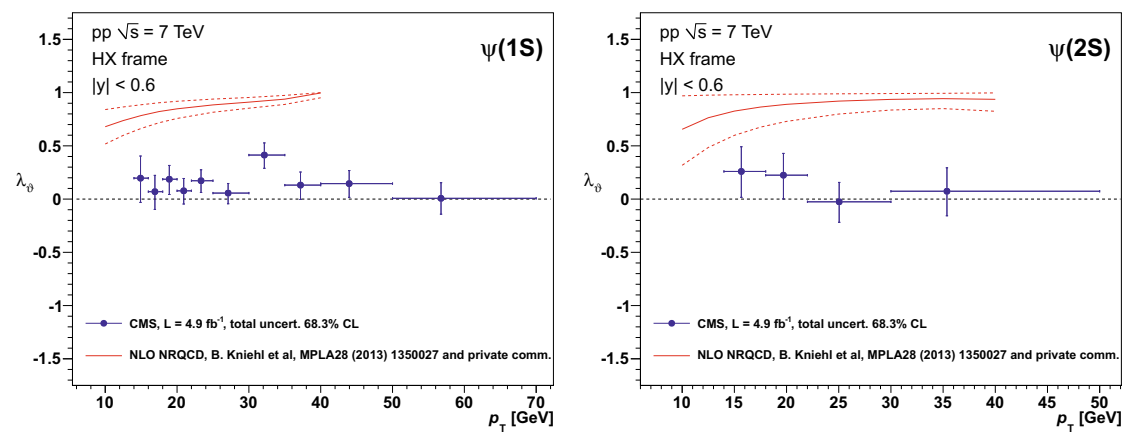

Figure 3. Comparison of the $J / \psi$ (left) and $\psi(2 S)$ (right) $\lambda_{\vartheta}$ parameter, measured in the $\mathrm{HX}$ frame as a function of the dimuon $p_{T}$, for the rapidity range $|y|<0.6$, to predictions for the directly produced mesons from NLO NRQCD.

where $\mathcal{N}(\vec{\lambda})$ is the normalization factor. This analysis uses the efficiency $\epsilon\left(\vec{p}_{1}, \vec{p}_{2}\right)$ as a function of the muon momentum, attributing to each event a probability dependent on the full event kinematics and on the values of the polarization parameters, without the need to assume any particular production kinematics.

Figure 2 shows an example of the measured $\cos \vartheta$ and $\varphi$ distributions.

None of the three polarization frames shows large polarizations and the measured values of $\lambda_{\vartheta}$ are in clear disagreement with the NLO NRQCD calculations (Fig. 3). While a small prompt $J / \psi$ polarization could be interpreted as smearing effect due to the decays of heavier (P-wave) charmonium states, this explanation cannot apply to the $\psi(2 S)$ state, unaffected by feed-down decays from heavier charmonia. 

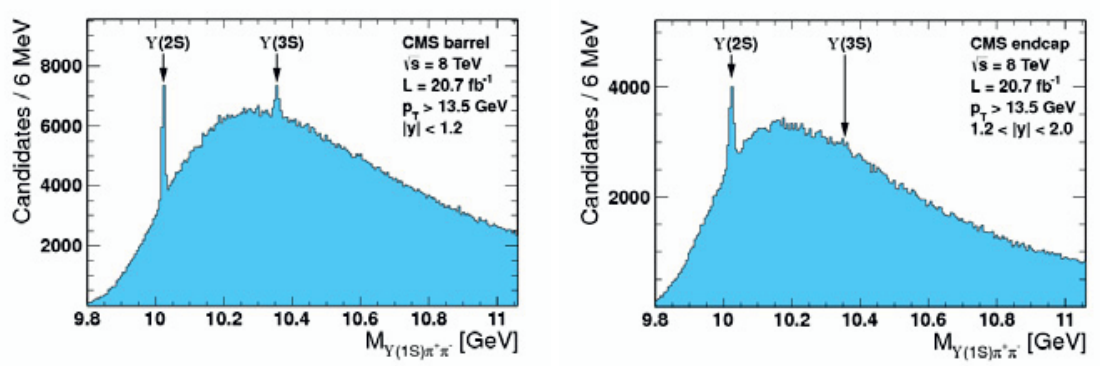

Figure 4. The reconstructed invariant mass distributions of the $\Upsilon(1 S) \pi^{+} \pi^{-}$candidates at mid (left) and forward (right) rapidities. A constraint on the dimuon invariant mass be equal to the world average $\Upsilon(1 \mathrm{~S})$ mass has been applied. Arrows indicate the world average $\Upsilon(2 S)$ and $\Upsilon(3 S)$.

\section{Search for a new bottomonium state decaying into $\Upsilon(1 S)$}

In the past few years, many unexpected charmonium states, such as the $\mathrm{X}(3872)$ and the $\mathrm{Y}(4260)$, have been discovered at the $B$-factories. Also, hints of bottomonium exotic resonances, like the $\mathrm{Y}_{b}$ candidate, have been found in the Belle experiment. The production rate for the $\mathrm{Y}_{b}$ state is expected to be very small at the LHC.

Considering that the $\mathrm{X}(3872)$ decays into $J / \psi \pi^{+} \pi^{-}$, a bottomonium counterpart, denoted as $\mathrm{X}_{b}$, would be expected to decay through $\mathrm{X}_{b} \rightarrow \Upsilon(1 \mathrm{~S}) \pi^{+} \pi^{-}$.

The CMS collaboration published a search for the $\mathrm{X}_{b}$ exotic bottomonium [2], through its decay $\mathrm{X}_{b} \rightarrow \Upsilon(1 \mathrm{~S}) \pi^{+} \pi^{-}$, using data from the $2012 \mathrm{pp}$ LHC run, corresponding to $20.7 \mathrm{fb}^{-1}$. Several known properties of the $\mathrm{X}(3872)$ state can provide clues in the search for the $\mathrm{X}_{b}$ resonance.

This analysis searches for a further peak, other than the known $\Upsilon(2 S)$ and $\Upsilon(3 S)$ mesons, in the $\Upsilon(1 S) \pi^{+} \pi^{-}$invariant-mass spectrum. The measurement is normalized through the decay $\Upsilon(2 \mathrm{~S}) \rightarrow \Upsilon(1 \mathrm{~S}) \pi^{+} \pi^{-}$, as a function of the $\mathrm{X}_{b}$ mass between 10 and $11 \mathrm{GeV}$, such that many systematic uncertainties cancel out.

Candidates are reconstructed from two identified muons and two additional charged tracks assumed to be pions. The kinematic region of the candidate investigated by this search is for $p_{T}>$ $13.5 \mathrm{GeV}$ and $|y|<2.0$. Since the mass resolution and the background level depend on the rapidity regions under study, data are divided into a "barrel" $(|y|<1.2)$ and an "endcap" $(1.2<|y|<2.0)$ region.

Figure 4 shows the reconstructed invariant-mass distributions. Except from the peaks corresponding to the known bottomonium resonances, the mass spectrum does not show any other outstanding structure. Unbinned maximum-likelihood fits are performed on the invariant mass distributions in steps of $10 \mathrm{MeV}$ to test the statistical significance of a hypothetical resonance. The relation between the $\mathrm{X}_{b}$ and the $\Upsilon(2 \mathrm{~S})$ yields is

$$
N_{\mathrm{X}_{b}}^{\mathrm{obs}}=R \times N_{\Upsilon(2 \mathrm{~S})}^{\mathrm{obs}} \times \frac{\epsilon_{\mathrm{X}_{b}}}{\epsilon_{\Upsilon(2 \mathrm{~S})}}
$$

where $N_{i}^{\text {obs }}$ are the observed yields and $\epsilon_{\mathrm{X}_{b}} / \epsilon_{\Upsilon(2 S)}$ is the ratio of the overall efficiencies.

Given no strong hint of a signal in the present data, an upper limit on $R$ is calculated. Systematic uncertainties are implemented as nuisance parameters in the likelihood and the final result is obtained combining the results from the two rapidity categories. 

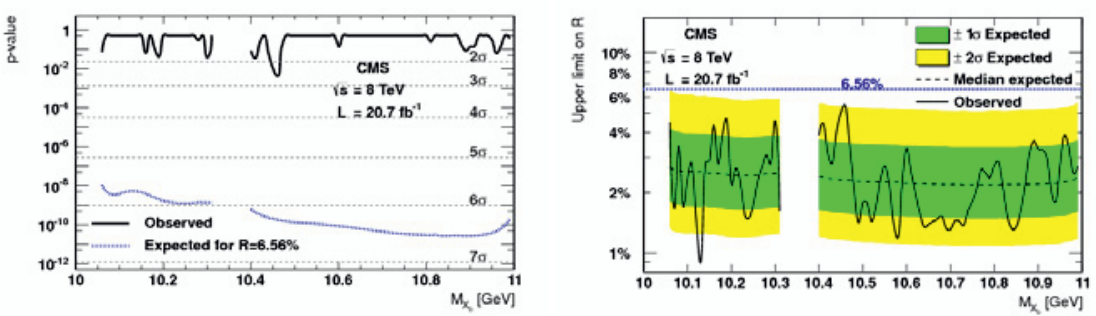

Figure 5. Left: The local $p$-values as a function of the injected $\mathrm{X}_{b}$ mass. The solid curve shows the observed local $p$-values, while the dashed curve shows the expected $p$-values if the relative signal strength to the normalization channel $R$ is equal to $6.56 \%$, that is the the ratio of $\mathrm{X}(3872)$ and $\psi(2 S)$. Right: Exclusion limits as a function of the exotic $\mathrm{X}_{b}$ mass, at $95 \% \mathrm{CL}$, on $R$. The solid curve shows the observed limits, while the dashed curve represents the limits expected for a pure background hypothesis.

Figure 5 (left) shows the observed local $p$-values. The smallest local $p$-value is at $10.46 \mathrm{GeV}$, corresponding to a statistical significance of $2.6 \sigma$, which is reduced to $0.8 \sigma$ when taking into account the "look- elsewhere effect". Instead, Figure 5 (right) shows the exclusion limits as a function of the $\mathrm{X}_{b}$ mass, at $95 \%$ confidence level (CL). The observed upper limits are in the range $0.9-5.4 \%$, depending on the assumed mass. These are the first upper limits on the production of a possible $\mathrm{X}_{b}$ at a hadron collider.

\section{Measurement of the $B_{s}^{0} \rightarrow \mu^{+} \mu^{-}$branching fraction and search for $B^{0} \rightarrow \mu^{+} \mu^{-}$decays}

The $B_{(s)}^{0} \rightarrow \mu^{+} \mu^{-}$rare decays are among the most promising investigation channels for the search of new physics. These decays proceed through SM penguin and box diagrams and are helicity suppressed; the SM predicts very small Branching Fractions (BFs): $3.5 \times 10^{-9}$ for the time-integrated $B_{s}^{0} \rightarrow \mu^{+} \mu^{-}$decay, and $1.1 \times 10^{-10}$ for the $B^{0} \rightarrow \mu^{+} \mu^{-}$decay. These tiny values and their small theoretical relative uncertainties (less than $10 \%$ ) make these decays very sensitive to many new physics scenarios.

The CMS experiment published its first measurement of the $B_{s}^{0} \rightarrow \mu^{+} \mu^{-}$BF and the most recent upper limit on $B^{0} \rightarrow \mu^{+} \mu^{-}$, performed with the full 2011 and $2012 \mathrm{LHC}$ data $\left(25 \mathrm{fb}^{-1}\right)$ [3].

The branching fraction is normalized to the $B^{ \pm} \rightarrow J / \psi K^{ \pm}$decay, to minimize uncertainties related to the $b \bar{b}$ production cross section and the integrated luminosity. Furthermore, many efficiency systematic uncertainties are reduced, using a decay channel with a signature similar to the signal decay. The measured $B_{s}^{0} \rightarrow \mu^{+} \mu^{-}$branching fraction, thus, is:

$$
\mathcal{B}\left(B_{s}^{0} \rightarrow \mu^{+} \mu^{-}\right)=\frac{N_{B_{s}^{0}}}{N_{B^{ \pm}}} \frac{f_{\mathrm{u}}}{f_{\mathrm{s}}} \frac{\varepsilon_{B^{ \pm}}}{\varepsilon_{B_{s}^{0}}} \mathcal{B}\left(B^{ \pm}\right)
$$

where $N_{i}$ is the number of reconstructed signal or normalization events, $\varepsilon_{i}$ is the total efficiency for the signal or the normalization decay, $\mathcal{B}\left(B^{ \pm}\right)$is the branching fraction for $B^{ \pm} \rightarrow J / \psi K^{ \pm} \rightarrow \mu^{+} \mu^{-} K^{ \pm}$ and $f_{\mathrm{u}} / f_{\mathrm{s}}$ is the ratio of the $B^{ \pm}$and $B_{s}^{0}$ fragmentation fractions.

The $B_{s}^{0} \rightarrow J / \psi \varphi$ decay is studied in data as a control channel to validate the Monte Carlo (MC) $B_{s}^{0}$ distributions. 

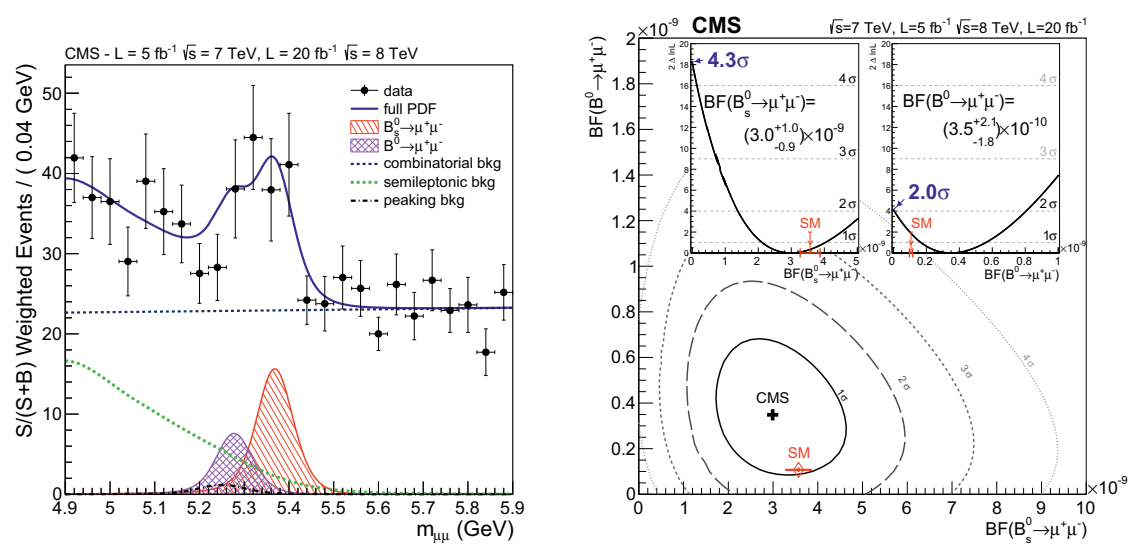

Figure 6. Left: Combination of all data categories for $B_{(s)}^{0} \rightarrow \mu^{+} \mu^{-}$. The individual categories are weighted with $S /(S+B)$, where $S(B)$ is the signal (background) determined at the $B_{s}^{0}$ peak position. Right: Scan of the ratio of the joint likelihood for $\mathcal{B}\left(B_{(s)}^{0} \rightarrow \mu^{+} \mu^{-}\right)$. As insets, the likelihood ratio scan for each of the branching fractions when the other is profiled together with other nuisance parameters; the significance at which the background-only hypothesis is rejected is also shown.

To avoid possible biases the invariant mass signal region is kept blind until all selection criteria are established. Multivariate studies, using boosted decision trees (BDT), are used to separate dimuon combinatorial background events, coming from separate weak $B$ decays, from signal events. Variables related to the secondary vertex, such as flight length, pointing angle, impact parameter and the isolation are included in the training. For the combinatorial background the BDT training utilizes events from the invariant mass side-bands, while MC simulations are used for the signal.

A clean muon identification is needed to reduce background events from rare two-body $B$ decays (such as $B_{s}^{0} \rightarrow K^{+} K^{-}$) that form the "peaking" background, and rare three-body $B$ decays (such as $B^{0} \rightarrow \pi^{-} \mu^{+} v_{\mu}$ ) that form the "semileptonic" background. All these events contain hadrons wrongly identified as muons, mainly due to punch-through or decays-in-flight. To reduce the "muon misidentification" rate, a further BDT analysis is performed on track-related quantities, such as the $\chi^{2}$ of the track global fit and the number of track hits, that present different distributions between true muons and misidentified muons. The training is performed with $\mathrm{MC} B_{s}^{0} \rightarrow \mu^{+} \mu^{-}$muons against $\mathrm{MC} B_{s}^{0} \rightarrow K^{+} K^{-}$ kaons, wrongly identified as muons.

An unbinned maximum likelihood fit on the invariant mass distribution is used to extract the signal and background yields. The dimuon mass distribution is divided into categories corresponding to different bins in the $B_{s}^{0}$ BDT parameter, that are fitted simultaneously.

An excess of $B_{s}^{0} \rightarrow \mu^{+} \mu^{-}$decays, consistent with the SM expectation, is observed above the background predictions, resulting in the measured branching fraction of $\mathcal{B}\left(B_{s}^{0} \rightarrow \mu^{+} \mu^{-}\right)=\left(3.0_{-0.9}^{+1.0}\right) \times$ $10^{-9}$. The significance of the excess is $4.3 \sigma$. Figure 6 on the left shows, as an illustrative purpose, the fit results on the weighted combined data. On the right, the ratio of the joint likelihood for $\mathcal{B}\left(B_{(s)}^{0} \rightarrow\right.$ $\left.\mu^{+} \mu^{-}\right)$is shown.

No significant excess is observed for the $B^{0} \rightarrow \mu^{+} \mu^{-}$decay, and an upper limit of $\mathcal{B}\left(B^{0} \rightarrow \mu^{+} \mu^{-}\right)<$ $1.1 \times 10^{-9}$ at $95 \%$ confidence level is determined with the $\mathrm{CL}_{\mathrm{S}}$ method (Fig. 7, left). 

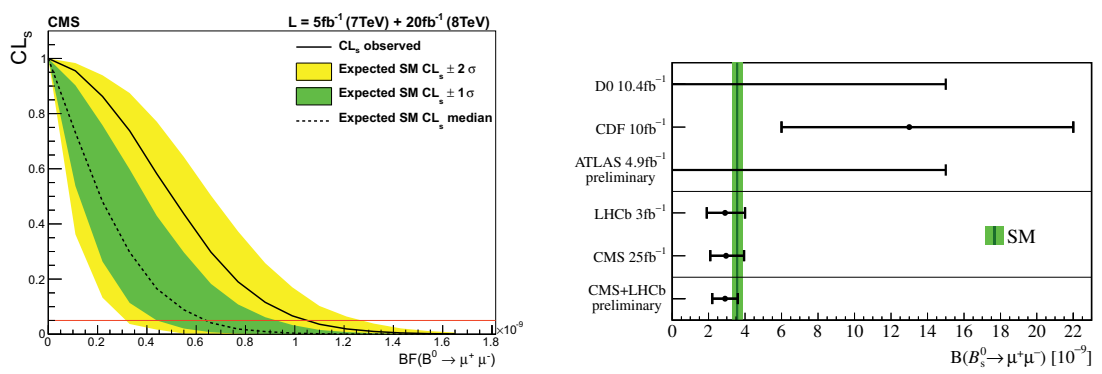

Figure 7. Left: Expected and observed $\mathrm{CL}_{\mathrm{S}}$ for $\mathcal{B}\left(B^{0} \rightarrow \mu^{+} \mu^{-}\right)$as a function of the assumed branching fraction. Right: Comparison of the results from D0, CDF, ATLAS, CMS and LHCb, the combined value, and the SM prediction (vertical line) for the time-integrated branching fraction $\mathcal{B}\left(B_{s}^{0} \rightarrow \mu^{+} \mu^{-}\right)$. The width of the vertical band represents the uncertainty in the SM prediction. The error bars represent the combined statistical and systematic uncertainties.

A first preliminary combination of the CMS measurements with the LHCb results was performed [4]. Taking into account the $100 \%$ correlation on $f_{\mathrm{s}} / f_{\mathrm{u}}$, results are

$$
\begin{aligned}
& \mathcal{B}\left(B_{s}^{0} \rightarrow \mu^{+} \mu^{-}\right)=(2.9 \pm 0.7) \times 10^{-9} \\
& \mathcal{B}\left(B^{0} \rightarrow \mu^{+} \mu^{-}\right)=\left(3.6_{-1.4}^{+1.6}\right) \times 10^{-10}
\end{aligned}
$$

which are also shown in Fig. 7, right. The combined significance of the $B_{s}^{0}$ signal reaches more than $5 \sigma$, while the $B^{0}$ significance still remains below $3 \sigma$.

\section{Conclusions}

In this talk I reported the most recent CMS $B$-physics results, in particular, focusing on the polarization of the prompt $J / \psi$ and $\psi(2 S)$ mesons, on the search for a new bottomonium state decaying into $\Upsilon(1 S)$ mesons and on the measurement of the $B_{s}^{0} \rightarrow \mu^{+} \mu^{-}$branching fraction and the $B^{0} \rightarrow \mu^{+} \mu^{-}$upper limit.

These represent the latest results of the CMS $B$-physics successful programme, thanks to the used flexible triggers, the efficient muon reconstruction and resolution and the accurate tracker vertexing of the CMS detector.

Several $B$ physics analyses are still ongoing, either studying new channels, or updating old results with full statistic. Many more details and results can be found at the CMS $B$-physics public page: https://twiki.cern.ch/twiki/bin/view/CMSPublic/PhysicsResultsBPH

\section{Acknowledgments}

The author wishes to thank the organizers of ICNFP2013 for the excellent organization and for the wonderful location.

\section{References}

[1] S. Chatrchyan et al. [CMS Collaboration], Phys. Lett. B 727 (2013) 381 [arXiv:1307.6070 [hepex]]. 
EPJ Web of Conferences

[2] S. Chatrchyan et al. [CMS Collaboration], Phys. Lett. B 727 (2013) 57 [arXiv:1309.0250 [hepex]].

[3] S. Chatrchyan et al. [CMS Collaboration], Phys. Rev. Lett. 111 (2013) 101804 [arXiv:1307.5025 [hep-ex]].

[4] CMS and LHCb Collaborations, CMS-PAS-BPH-13-007, CERN-LHCb-CONF-2013-012. 